\title{
Overlap Interfaces in Hierarchical Spin-Glass models
}

\author{
Silvio Franz ${ }^{1}$, T Jörg ${ }^{1}$ and Giorgio Parisi ${ }^{2}$ \\ ${ }^{1}$ LPTMS, UMR 8626 CNRS et Université Paris-Sud, Bâtiment 100, 91405 Orsay \\ CEDEX, France \\ ${ }^{2}$ Dipartimento di Fisica and INFM-CNR, Università di Roma "La Sapienza", \\ Piazzale Aldo Moro 2, 00185 Roma, Italy
}

\begin{abstract}
We discuss interfaces in spin glasses. We present new theoretical results and a numerical method to characterize overlap interfaces and the stability of the spinglass phase in extended disordered systems. We use this definition to characterize the low temperature phase of hierarchical spin-glass models. We use the Replica Symmetry Breaking theory to evaluate the cost for an overlap interface, which in these models is particularly simple. A comparison of our results from numerical simulations with the theoretical predictions shows good agreement.

PACS numbers: 05.10.-a,05.50.+q,75.10.Nr,75.40.Mg
\end{abstract}

\section{Introduction}

The study of interfaces is often used to investigate the stability of ordered coexisting phases. Low temperature ordered phases are spatially homogeneous, however, interfaces can be induced by forcing heterogeneous boundary conditions [1]. For example, in the case of phases characterized by different values of an order parameter, one can fix the order parameter at the boundaries along one selected direction to two different values. For stable phases the induced interface costs an amount of free-energy proportional to a power of the interface size. At the lower critical dimension this power vanishes and long-range order becomes unstable.

In spin glasses, due to the lack of a physical order parameter allowing to distinguish the different phases, this definition of an interface does not seem of practical use. Different proposals have been put forward to define interfaces in spin glasses. A common procedure [2, 3, 4], involves the comparison of free-energies between systems with periodic and antiperiodic boundary conditions, respectively. This leads to a definition of an interface exponent, from the relation: $\left|F_{\text {per }}-F_{\text {anti-per }}\right| \sim L^{\theta}$ which plays a fundamental rôle in the droplet picture of spin glasses [4, 5]. Measures of $\theta$ have been used to perform a high-precision estimate of the spin-glass lower critical dimension $D_{C} \approx 2.5$ in Ref. [6] (defined as the value of $D$ for which the interface exponent $\theta$ vanishes). An alternative definition [7] uses the free-energy cost that is needed to impose a spatial heterogeneity in the order parameter, which in spin glasses is the overlap, i.e. 
a measure of the similarity between two equilibrated configurations of the same system [8]. One needs to consider two identical copies of the system constrained in such a way to have their mutual overlaps on the boundaries fixed to some preassigned values. This definition is well suited to test an important property emerging in the Replica Symmetry Breaking (RSB) of the spin-glass phase: while the probability distribution $p(q)$ for the overlap is broadt, reflecting the presence of many different and almost degenerate equilibrium phases, one has that for any couple of equilibrated configurations their mutual overlap is spatially homogeneous on a large scale. Two configurations with a given value of the overlap in a given macroscopic portion of space will have the same overlap everywhere.

Starting from this observation a pair of identical systems subject to "twisted overlap" boundary conditions was considered in [7]. Two different values $q_{1}$ and $q_{2}$ of the overlap were imposed on the two boundaries along a selected direction, chosen among the ones with non-zero probability density in the $p(q)$. It was then argued, that if RSB is present, the boundaries induce a smooth overlap profile in space that interpolates between the two values. Above the lower critical dimension $D_{c}$ for spin-glass order this has a diverging free-energy cost, and one can expect the following scaling with $L$ and $\left|q_{1}-q_{2}\right|$ :

$$
\Delta \tilde{F}\left(q_{1}, q_{2}\right) \sim L^{\theta_{q}}\left|q_{1}-q_{2}\right|^{b_{q}}
$$

where the exponent $\theta_{q}=D-D_{c}$ is positive for $D>D_{c}$. A mean-field calculation of $b_{q}$ and the interface exponent $\theta_{q}$ gave the non-trivial values of $b_{q}=5 / 2$ and $D_{C}=5 / 2$. Remarkably, while measuring a quite different property, the resulting value of the lower critical dimension is in very good agreement with the one estimated by the periodicantiperiodic boundary condition method [4, 6].

Unfortunately, despite its potentially informative content on the nature of the spinglass phase, the definition of [7] was never used in numerical studies of the spin-glass phase. This is probably due to the difficulty of imposing values of the overlap at the boundaries.

In this paper we start from the general observation, in fact not at all specific to spin glasses, that it is possible to induce interfaces with an alternative procedure. One can divide the physical system into two contiguous halves and impose fixed values to the bulk order parameters in each of the two halves. This procedure, theoretically equivalent to fixing heterogeneous boundary conditions, is better suited for numerical investigations, since the resulting free-energy cost can be related to the probability of a spontaneous fluctuation in an unconstrained system.

In the case of spin glasses we have to consider two real replicas and impose values $q_{1}$ and $q_{2}$ of their mutual overlaps in two contiguous half spaces. This way of imposing heterogeneity in the system induces an overlap profile in space equal to the one induced by fixing the overlap on the boundaries to suitable values $q_{1}^{\prime}$ and $q_{2}^{\prime}$. One then has a

$\ddagger$ This is in contrast to the droplet picture of the spin-glass phase where the distribution $p(q)$ - although having noticeable tails for finite systems - reduces to two delta functions in the thermodynamic limit. 
free-energy cost $\Delta F\left(q_{1}, q_{2}\right)$ of the form (11) with the same exponents $\theta_{q}$ and $b_{q}$ \& The freeenergy cost for imposing the different overlaps is related by Boltzmann's relation to the probability that a spontaneous fluctuation of a couple of unconstrained systems produce the values $q_{1}$ and $q_{2}$ of the overlaps in the two half spaces: $P_{L}\left(q_{1}, q_{2}\right)=e^{-\beta \Delta F\left(q_{1}, q_{2}\right)}$, which is a large deviation formula. This quantity has the advantage that it is easily accessible in numerical simulations and therefore we base our analysis on it.

In this paper we focus our attention on overlap interfaces in Dyson-like hierarchical spin-glass models.

Hierarchical models without disorder [9] have played an important rôle in the theoretical and mathematical understanding of critical points [10, 11]. In the ferromagnetic Dyson model one can write exact renormalization group equations that involve the iteration of a function of a single variable (for a review see [12]). Hierarchical spin-glass models of the Dyson kind have not to our knowledge been considered in the literature and are in our opinion very attractive as they could allow for an analytical study of non-mean-field disordered critical points and nontrivial low temperature phases. These models provide a hierarchical version of spin glasses with power-law interactions introduced in [13]. One-dimensional models with power-law interactions have recently received a great deal of attention as test grounds for theoretical ideas about finite-range spin-glass phases [14, 15, 16, 17, 18]. Here, following Dyson, we propose to use the tree topology as a further simplification. We choose to start the study of these models focusing on interface properties, for which we can get particularly simple theoretical predictions in the RSB framework. We study the system through the replica method, deriving a recursion equation relating the replica partition functions at the different levels of the hierarchy. This relation, which in principle codes for all the thermodynamic properties of the system is analyzed in a self-consistent way to obtain the interface freeenergy. We test our prediction in numerical simulations, finding good agreement.

The organization of the paper is the following: in section 2 we discuss the definition of the interfaces and how in principle interfaces can be evaluated and some expectations based on RSB theory. Section 3 is devoted to the definition of the models we use. In section 4 we discuss possible scenarios and expectations and in section 5 we sketch the theoretical derivation of the interface free-energy cost in hierarchical models. Section

6] discusses the numerical simulations. Finally, section 7 concludes the paper. In the appendix we discuss the numerical characterization of the critical point.

\section{Generalities}

In this section we define the probability distribution of overlaps in contiguous half spaces and discuss how it can be computed in principle in the large deviation regime. For definiteness we consider a spin-glass system with Ising spins $S_{i}= \pm 1$ on a set of indices

$\S$ This is strictly true for a couple of systems with open boundary conditions where the first procedure is naturally defined. The second procedure has the advantage of being defined also for periodic boundary conditions. 
$i \in \Lambda$ with $N$ elements, that we divide in two "half spaces" $\Lambda_{1}$ and $\Lambda_{2}$ with $N / 2$ elements each. For two spin configurations $\mathbf{S}$ and $\mathbf{S}^{\prime}$ one can define the partial overlaps $Q_{r}\left(S, S^{\prime}\right)$ in each of the half spaces $\Lambda_{r}, r=1,2$ as $Q_{r}\left(S, S^{\prime}\right)=1 /(N / 2) \sum_{i \in \Lambda_{r}} S_{i} S_{i}^{\prime}$. The joint probability distribution function (PDF) of the two overlaps, for fixed value of the system size $L$ and quenched disorder $J$, is given by

$$
P_{J, L}\left(q_{1}, q_{2}\right)=\frac{1}{Z^{2}} \sum_{S, S^{\prime}} e^{-\beta H(S)-\beta H\left(S^{\prime}\right)} \delta\left(Q_{1}\left(S, S^{\prime}\right)-q_{1}\right) \delta\left(Q_{2}\left(S, S^{\prime}\right)-q_{2}\right),
$$

where $Z$ denotes the partition function. From this relation one gets the usual PDF of the total overlap by simple integration. One can consider the average over the disorder of (2), however, here we will concentrate on the large deviation regime, where one can expect

$$
P_{J, L}\left(q_{1}, q_{2}\right) \sim e^{-L^{\theta} \Delta_{F_{J}}\left(q_{1}, q_{2}\right)}
$$

with a positive $\Delta F_{J}\left(q_{1}, q_{2}\right)$. The exponent $L^{\theta_{q}} \Delta F_{J}\left(q_{1}, q_{2}\right)$ represents an interface freeenergy cost to maintain the constrained values. In this regime, the large deviation functional can be expected to be self-averaging and one needs to compute the average free-energy

$$
E \log \left(P_{J, L}\left(q_{1}, q_{2}\right)\right)=-L^{\theta_{q}} \Delta F\left(q_{1}, q_{2}\right) .
$$

The RSB implies that if one chooses $q_{1}=q_{2}$ in the domain where $p(q)$ is non-zero, then $\Delta F\left(q_{1}, q_{1}\right)=0$. The property of homogeneity of the overlap should then translate in a form for $\Delta F\left(q_{1}, q_{2}\right)$ of the kind $\Delta F\left(q_{1}, q_{2}\right)=g\left(\left(q_{1}+q_{2}\right) / 2\right)\left|q_{1}-q_{2}\right|^{b_{q}}$.

From the complete knowledge of the joint distribution (3) one can in principle extract the marginal distribution of the difference $u=q_{1}-q_{2}$

$$
P_{L}(u)=\int d q_{1} d q_{2} \delta\left(u-\left(q_{1}-q_{2}\right)\right) P_{L}\left(q_{1}, q_{2}\right) .
$$

In the large deviation regime, where this analysis is supposed to be valid, we have $\left|q_{1}-q_{2}\right|=O(1)$ for $L \rightarrow \infty$ and hence the integral should be dominated by the value of $q=\left(q_{1}+q_{2}\right) / 2$ that maximizes the function $g$. As a consequence $P_{L}(u)$, the PDF of $u$, should behave as $e^{-g^{*} L^{\theta_{q}}|u|^{b q}}$ in the tails. In order to understand the behavior for "small" values of $u$ we can suppose a smooth cross-over between the small and the large fluctuation regimes. In this case the form (44) suggests a finite probability for $\left|q_{1}-q_{2}\right|=O\left(L^{-\theta_{q} / b_{q}}\right)$, i.e., a scaling form $P_{L}\left(q_{1}, q_{2}\right)=H\left(\left(q_{1}+q_{2}\right) / 2,\left(q_{1}-q_{2}\right) L^{\theta_{q} / b_{q}}\right)$ and the marginal probability of the difference $u=q_{1}-q_{2}, P_{L}(u)=P\left(u L^{\theta_{q} / b_{q}}\right) L^{\theta_{q} / b_{q}}$. This quantity $P_{L}(u)$ is particularly easily accessible in numerical simulations and contains the the information on the ratio between the interface exponents. The comparison of this method with the study of the overlap correlation function [28], as well as the comparison of our analytic results in section 5 with pertubative calculations [27] will be not attempted in this paper. 


\section{Hierarchical Spin Glasses}

Here we introduce hierarchical spin-glass models on Dyson lattices. We will consider two families of models, a first one that is better suited for analytic studies and a second one that is more adapted to numerical simulations. In both families the spins are associated to the leaves of a binary tree, see fig. (11). The distance between two spins that, rising up in the hierarchy, meet after $l$ branches is naturally defined as $2^{l}$. The first family of models we would like to define, is the natural Spin-Glass generalization of the Ferromagnetic Dyson model ([9]). The Hamiltonian can be constructed iteratively, connecting the two non-interacting systems of $2^{k}$ spins $(k \in \mathbb{N})$ to form a composite system of $2^{k+1}$ spins in the following way:

$$
\begin{aligned}
H_{k+1}^{J}\left[S_{1}, \ldots, S_{2^{k+1}}\right] & =H_{k}^{J_{1}}\left[S_{1}, \ldots, S_{2^{k}}\right]+H_{k}^{J_{2}}\left[S_{2^{k}+1}, \ldots, S_{2^{k+1}}\right]- \\
& -\frac{1}{2^{(k+1) \sigma}} \sum_{i<j}^{1,2^{k+1}} J_{i j} S_{i} S_{j}
\end{aligned}
$$

having defined the Hamiltonian for a system at the first level of the hierarchy

$$
H_{1}^{J}\left(S_{1}, S_{2}\right)=-\frac{1}{2^{\sigma}} J S_{1} S_{2} .
$$

The couplings $J_{i j}$ are independent and identically distributed Gaussian random variables with zero mean and unit variance. The family is parametrized by the value of $\sigma$ that tunes the decrease of the strength of the interaction with the distance. The interaction strenght decrease as a power of the distance, the model is therefore a hierarchical counterpart of the one-dimensional spin glass with power-law interactions [13] which has received attention recently [14, 15, 16, 17, 18. As in this case, the model is defined for $\sigma \geq 1 / 2$. Exactly at the value $\sigma=1 / 2$ it reduces to the infinite range model [19]. Notice that the sum of the squares of the interaction terms that couple the two subsystems $\frac{1}{2^{(k+1) 2 \sigma}} \sum_{i<j}^{1,2^{k+1}} J_{i j}^{2}$ scales as $2^{2(k+1)(1-\sigma)}$. This is on the order of the volume for the mean-field value $\sigma=1 / 2$ and smaller than the volume for the nontrivial regime $\sigma>1 / 2$.

The second family we consider is a dilute version of model (6) . We consider a Hamiltonian for $2^{k}$ spins with a fixed number of terms $P=\alpha 2^{k}(\alpha>1)$

$$
H_{k}^{J}\left[S_{1}, \ldots, S_{2^{k}}\right]=-\sum_{\mu=1}^{P} J^{\mu} S_{i^{\mu}} S_{j^{\mu}},
$$

where the $J^{\mu}$ are taken as \pm 1 variables with equal probability, and we choose the interacting couples of sites $\left(i^{\mu}, j^{\mu}\right)$ independently term by term and in a way that, if the distance between $i^{\mu}$ and $j^{\mu}$ on the binary tree with $2^{k}$ branches is $2^{n}$, we put the coupling with a probability given by

$$
P\left(i^{\mu}, j^{\mu}\right)=A_{k} 2^{n(1-2 \sigma)},
$$

where $A_{k}$ is a constant chosen such that it normalizes the probability. For $\sigma=1 / 2$ the model reduces to the Viana-Bray spin-glass model [20] on Erdös-Rényi random 


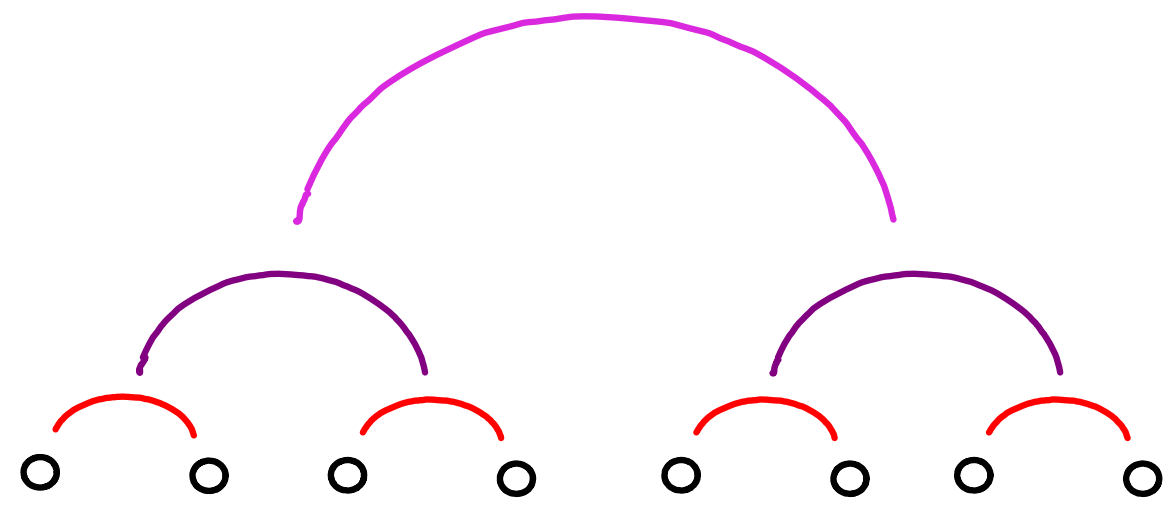

Figure 1. In our models the spins are associated to the leaves of a binary tree with $k$ generation. In the first model all the spins interact with each other with a strength that decreases as a power of the distance. In the second case, each spin interacts directly with a small number of other spins at random, with a probability of interaction that behaves as a decreasing power of the distance.

graph. We will refer to the two models defined above as model I (66) and model II (8)), respectively. Both models should belong to the same universality class for the same value of $\sigma$, with respect to the critical and the low temperature properties. For $\sigma \in[1 / 2,1]$ the models have a finite temperature spin-glass transition. The critical point has a classical (i.e., Gaussian) character for $\sigma \in[1 / 2,2 / 3]$, while it has a non-classical character for $\sigma \in[2 / 3,1]$. As observed in [18, 21], diluted models as the one in (8) are convenient in numerical simulations since the number of interactions for each spin does not grow with the system size. Spending an equal amount of computational effort we can therefore study much bigger sizes than for model I, hoping that the finite-size corrections are comparable in both models.

\section{Interfaces in hierarchical models}

Let us discuss some scenarios for the behavior of the PDF $P_{L}\left(q_{1}, q_{2}\right)$ corresponding to different possible physical situations.

The simplest physical situation is the paramagnetic state where long-range order is absent. In this case we have a finite correlation length $\xi$ and for large $k$ one can expect $q_{1}$ and $q_{2}$ to be sums of $\sim L / \xi$ independent terms. The resulting probability distribution of $q_{1}$ and $q_{2}$ is a product of two independent Gaussians with variance proportional to $\xi / L$.

We can then consider a condensed phase with only two pure states. In this case, the space average value of the overlap can take two values $\pm q^{*}$. Small fluctuations will still be Gaussian as in the paramagnetic case. Due to the symmetry of the model, where groups of spins at a given level of the hierarchy are on the same foot, one can expect that large fluctuations where $q_{1} \approx q^{*}$ and $q_{2} \approx-q^{*}$ (or vice versa) will imply a free-energy cost $\Delta F=A 2^{(k+1) 2(1-\sigma)}$ and have a probability $e^{-\beta A 2^{(k+1) 2(1-\sigma)}}$.

The most interesting possibility is a spin-glass phase with RSB. In this case one 
has a zero mode in the free-energy associated to the existence of a couple of states with overlaps taking values in a finite interval [8]. If one chooses $q_{1}=q_{2}=q$ one finds a broad distribution $p_{k+1}(q)$, which for large $k$ is close to the limiting distribution $p(q)$. Consider first a system in absence of interactions at the $k+1$-th level. The two subsystems are independent and $P_{k+1}^{(0)}\left(q_{1}, q_{2}\right)=p_{k}\left(q_{1}\right) p_{k}\left(q_{2}\right)$. If the interaction is switched on, we can expect a free-energy cost $\Delta F\left(q_{1}, q_{2}\right)=g\left(q_{1}+q_{2}\right) 2^{2(1-\sigma) k}\left|q_{1}-q_{2}\right|^{b_{q}}$. The overlap interface exponent is just given by $\theta_{q}=2(1-\sigma)$ due to the fact that the total interaction strength squared between the two parts scales as $2^{2(1-\sigma) k}$. The value of the exponent $b_{q}$ as well as the function $g$ can be computed supposing RSB at the level $k$. The detailed calculation is quite involved and is presented in the next section; here we just give the net result, valid in the regime $q_{1}, q_{2} \in\left[-q_{E A}, q_{E A}\right]$. Neglecting prefactors we could not compute, it reads:

$$
P_{k+1}\left(q_{1}, q_{2}\right) \sim e^{-p\left(\left(q_{1}+q_{2}\right) / 2\right) 2^{2(1-\sigma)(k+1)}\left|q_{1}-q_{2}\right|^{3}} .
$$

Notice the appearance of the $k \rightarrow \infty$ function $p(q)$ in the exponent of (10). From this formula one can extract the conditional probability of the difference $u=q_{1}-q_{2}$ for a fixed value of the semi-sum $q=\left(q_{1}+q_{2}\right) / 2$ :

$$
P_{k+1}(u \mid q) \sim e^{-p(q) 2^{2(1-\sigma)(k+1)}|u|^{3}} .
$$

Equation (11) summarizes our prediction for the hierarchical model. For a fixed value of the sum, the difference of the overlap is distributed according to the exponential of the cube, which is different from a naive Gaussian guess. The coefficient of the exponential is equal to the function $p(q)$ which can be evaluated in independent measurements. In numerical simulations it might be complicated to collect sufficient statistics to condition $u$ to the value of $q$. One can then turn to the unconditional distribution. In the large deviation regime, where $u \sim O(1)$, this should be dominated for large $k$ and finite $u$ by the value of $q$ which maximizes (11). For functions $p(q)$ as the one commonly met in spin-glass systems this is the value $q=q_{E A}$ which is the largest possible value of the overlap in the thermodynamic limit.

The form (11) suggests that the order of magnitude of the typical fluctuations is $|u| \sim 2^{2 / 3(1-\sigma)(k+1)}$. In this regime, the unconditional distribution of $u$ involves the convolution of (111) with a presently unknown prefactor and cannot be computed. All the form (11) tells us for the unconditioned distribution in this regime is that the finite volume distribution of $u$ admits the scaling form

$$
P_{k+1}(u)=\int d q P_{k+1}(u \mid q) P_{k+1}(q)=W\left(2^{2 / 3(1-\sigma)(k+1)}|u|\right) 2^{2 / 3(1-\sigma)(k+1)} .
$$

In order to compute the scaling function $W$ the knowledge of the prefactor in (11) would be necessary. We remark that the exponent in this scaling is a strong consequence of RSB theory, a naive guess would have suggested a Gaussian distribution with scaling variable $2^{2(1-\sigma)(k+1)} u^{2}$. 


\section{Replica derivation of formula (11)}

We analyze model I through the replica method. In order to compute the free-energy, it is natural to consider a recursion that relates the average partition function of $n$ replicas $S_{i}^{a}, a=1, \ldots, n$, with fixed mutual overlaps $Q_{a b}=\frac{1}{2^{k}} \sum_{i=1}^{2^{k}} S_{i}^{a} S_{i}^{b}$. Defining

$Z_{k}[\mathbf{Q}]=E_{J}\left[\sum_{\mathbf{S}} \exp \left(-\sum_{a=1}^{n} H_{k}^{J}\left(S_{1}^{a}, \ldots, S_{2^{k}}^{a}\right)\right) \prod_{a<b}^{1, n} \delta\left(Q_{a b}-\frac{1}{2^{k}} \sum_{i=1}^{2^{k}} S_{i}^{a} S_{i}^{b}\right)\right]$,

where $E_{J}$ denotes the average over the disorder, we can write:

$Z_{k}[\mathbf{Q}]=\exp \left(\frac{\beta^{2}}{4} 2^{2(1-\sigma) k} \operatorname{Tr} \mathbf{Q}^{2}\right) \int \mathcal{D} \mathbf{Q}_{1} \mathcal{D} \mathbf{Q}_{2} Z_{k-1}\left[\mathbf{Q}_{1}\right] Z_{k-1}\left[\mathbf{Q}_{2}\right] \delta\left(\mathbf{Q}-\frac{\mathbf{Q}_{1}+\mathbf{Q}_{2}}{2}\right)$

For integer $n$ this is an exact relation based on the independence of the Hamiltonians of the sub-systems at level $k$. In principle, the thermodynamical properties of the system are encoded in this recursion and in its analytic continuations for $n \rightarrow 0$. For example it can be used to set up an epsilon expansion for the calculation of the critical indexes for $\sigma \in[2 / 3,1][22$. In this paper we just use (14) to study the probability distribution (11). This can be done with the technique of constrained replicas, introduced and discussed at length in [7]. In order to consider constrained free-energies for two replicas with fixed overlaps one should fix some of the elements of the matrix $Q_{a b}^{r} r=1,2$ to the values of the constraints. Writing $n=2 n^{\prime}$ the constraint reads $Q_{a, a+n^{\prime}}^{r}=Q_{a+n^{\prime}, a}^{r}=q_{r}$ for $r=1,2$ and $a=1, \ldots, n^{\prime}$. Introducing the replica free-energy at level $k$ for fixed $q_{1}$ and $q_{2}, Z_{k}\left[\mathbf{Q} \mid q_{1}, q_{2}\right]=e^{-\beta 2^{k} F_{k}\left[\mathbf{Q} \mid q_{1}, q_{2}\right]}$ we see that one needs in principle to compute:

$$
\begin{aligned}
\int \mathcal{D} \mathbf{Q} e^{-\beta 2^{k} F_{k}\left[\mathbf{Q} \mid q_{1}, q_{2}\right]}= & \int \mathcal{D} \mathbf{Q}_{1} \mathcal{D} \mathbf{Q}_{2} e^{\left(\frac{\beta^{2}}{4} 2^{2(1-\sigma) k} \operatorname{Tr}\left(\mathbf{Q}_{1}+\mathbf{Q}_{2}\right)^{2}\right)} e^{-\beta 2^{k}\left(F_{k}\left[\mathbf{Q}_{1}\right]+F_{k}\left[\mathbf{Q}_{2}\right]\right)} \times \\
& \times \prod_{a=1}^{n^{\prime}} \delta\left(Q_{a, a+n^{\prime}}^{1}-q_{1}\right) \delta\left(Q_{a, a+n^{\prime}}^{2}-q_{2}\right) .
\end{aligned}
$$

This form suggests that for large $k$, supposing the knowledge about $F_{k}[\mathbf{Q}]$, the integral over $\mathbf{Q}_{1}$ and $\mathbf{Q}_{2}$ can be performed by the saddle-point approximation. Notice that the interaction term is sub-extensive and scales as $2^{2(1-\sigma)(k+1)}$, while the partial free-energies scale as the volume $2^{k}$. The interaction term does therefore not contribute to the saddle point, and the maximization with respect to the matrices $Q^{r}$ can be performed separately in each of the two sub-systems.

The value of the interaction term at the saddle point determines the free-energy difference $\Delta F\left(q_{1}, q_{2}\right)$.

We now study the consequences of the hypothesis that there is RSB in the system. Specifically, we suppose that in absence of any constraints RSB is described by a continuous Parisi function $q_{F}(x)$ taking values between the two extremes $-q_{E A}$ and $q_{E A}$. In the constrained problem, correspondingly, each of the matrices $Q^{r}$ is parametrized by two functions $q^{r}(x)$ and $p^{r}(x)$ with $x \in[0,1]$. As implied by the analysis in [7] the free-energy in each of the two sub-systems is then independent of $q^{r}$, and the function 
$q^{r}(x)$ and $p^{r}(x)$ can be directly related to the function $q_{F}(x)$ of the unconstrained system by the relations

$$
q(x)=\left\{\begin{array}{cc}
q_{F}(2 x) & x \leq x_{1} / 2 \\
q_{r} & x_{r} / 2<x \leq x_{r} \\
q_{F}(x) & x>x_{1}
\end{array} \quad p_{x}(u)=\left\{\begin{array}{cc}
q_{F}(2 x) & x \leq x_{r} / 2 \\
q_{r} & x>x_{r} / 2,
\end{array}\right.\right.
$$

where $x_{r}$ is the value of $x$ such that $q_{F}\left(x_{r}\right)=q_{r}$. We then see that the free-energy difference from the unconstrained case is entirely due to the interaction term, which can be evaluated using the saddle-point value of the matrices $Q_{1}$ and $Q_{2}$ :

$$
\operatorname{Tr}\left(\frac{\mathbf{Q}_{1}+\mathbf{Q}_{2}}{2}\right)^{2}=n\left[\left(\frac{q_{1}+q_{2}}{2}\right)^{2}-\int d x\left(\frac{q_{1}(x)+q_{2}(x)}{2}\right)^{2}+\left(\frac{p_{1}(x)+p_{2}(x)}{2}\right)^{2}\right]
$$

Substitution of (16) leads to

$$
\operatorname{Tr}\left(\frac{\mathbf{Q}_{1}+\mathbf{Q}_{2}}{2}\right)^{2}=n\left(-2 \int_{0}^{1} d x q_{F}(x)^{2}+\int_{x_{1}}^{x_{2}}\left(q_{2}-q_{F}(x)\right)\left(q_{F}(x)-q_{1}\right)\right),
$$

where without loss of generality we have supposed $q_{1}<q_{2}$. The first term is just the contribution that can be expected if $q_{1}=q_{2}$. Together with the two subsystems' freeenergy it just gives the free-energy of the system at the level $k+1$. The second term is associated to the free-energy excess needed to impose $q_{1} \neq q_{2}$. For small $q_{2}-q_{1}$ we can expand this last term and find

$$
n x^{\prime}\left(\frac{q_{1}+q_{2}}{2}\right)\left(q_{2}-q_{1}\right)^{3},
$$

where $x(q)$ is the inverse function of $q_{F}(x)$. Inserted in (15) and identifying $x^{\prime}(q)$ with $p(q)$ as discussed in length in [8] leads to (11)

$$
P_{k+1}\left(q_{1}, q_{2}\right) \sim e^{-p\left(\left(q_{1}+q_{2}\right) / 2\right) 2^{2(1-\sigma)(k+1)}\left|q_{1}-q_{2}\right|^{3}} .
$$

\section{Numerical Simulations}

In this section we discuss the results of numerical simulations used to test the behavior of the probability distribution of the overlap difference in the two subsystems. In order to deal with PDF's of a single variable we concentrated to the unconditional probability for which we have the theoretical prediction (12). We did not try to test the more detailed prediction (11) which would need the numerical determination joint PDF's of two variables.

We have simulated model II for $\alpha=3 / 2$, using parallel tempering to thermalize the system at low temperatures.

We have tested our theoretical predictions concentrating on two values of $\sigma$ : $\sigma=0.576$ which lies in the classical region $1 / 2<\sigma<2 / 3$, where the spin-glass transition is well described by mean-field theory, and $\sigma=0.707$ which lies in the nonclassical region $2 / 3<\sigma<1$, where the exponents are nontrivial. 


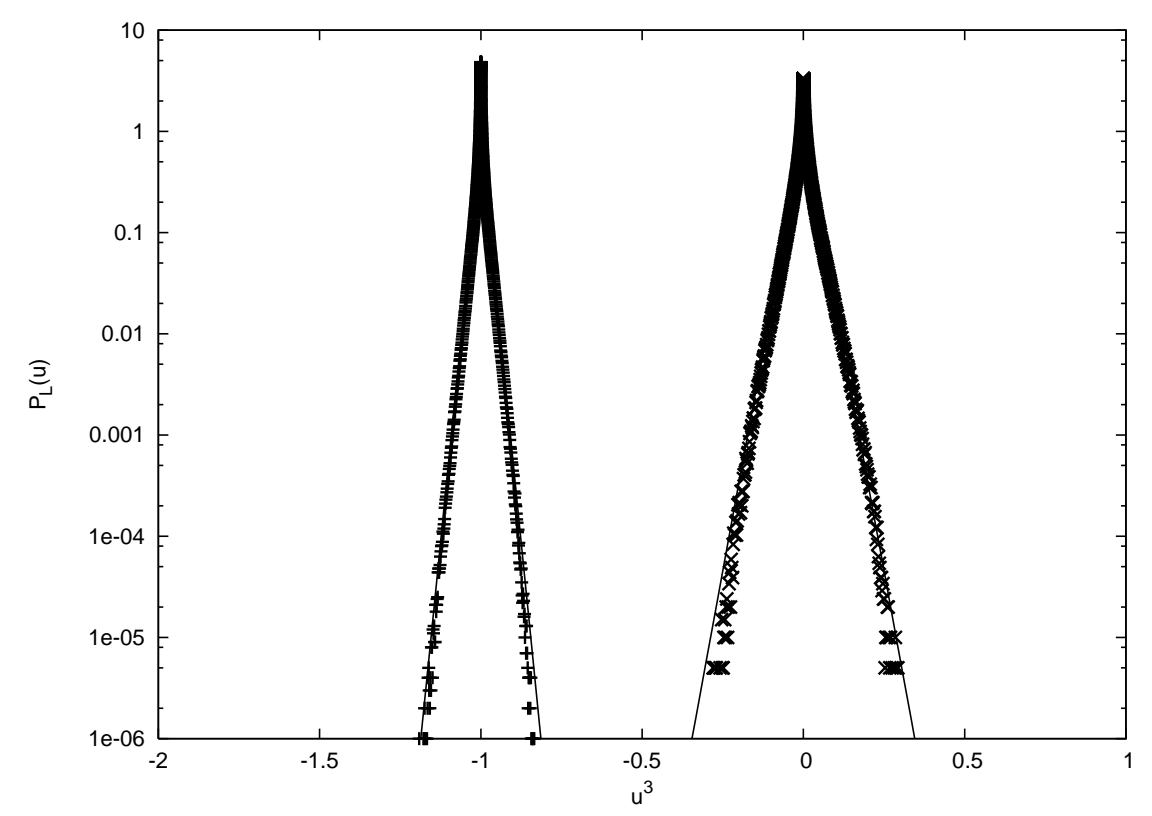

Figure 2. Probability distribution function $P_{k}(u)$ for $K=10, \sigma=0.576$ (left) in the classical region and $\sigma=0.707$ (right) in the non-classical region, plotted in a loglinear scale as a function of $u^{3}$. Both systems are in the low temperature phase, the temperatures being respectively $T=0.745$ and $T=0.740$. The data for $\sigma=0.576$ have been shifted of one unity to the right. For comparison we plot curves of the kind $a e^{b u^{3}}$ with $a$ and $b$ adjusted to fit the tails of the curves.

We have characterized the critical point of the model following the procedure that we describe in the appendix. We estimate the critical temperatures to be equal to $T_{c}=1.24(1)$ for $\sigma=0.576$ and $T_{c}=1.14(1)$ for $\sigma=0.707$.

To test the predictions described in the previous sections we measured the distribution of the variables $q$ and $u$ as a function of temperature and system size. We considered systems sizes of $2^{7}, 2^{8}, 2^{9}$ and $2^{10}$ spins. Averages were performed over 4000 samples for the smaller systems and 700 samples for the largest system. The configurations are thermalized during the first $2^{20}$ Monte Carlo (MC) sweeps of the runs and then data is collected for the subsequent $2^{20} \mathrm{MC}$ steps.

The first prediction we test is the validity of the cube-exponential form in the tails for large $u$. This is well observed in all our simulations. A typical example of our findings is depicted in figure (2) where we plot the function $P_{k}(u)$ for $k=10$ for the two different values of $\sigma$ in the classical and non-classical regime, respectively. The data are plotted together with functions of form $f(x)=a e^{-b|x|^{3}}$ which should be considered as a guide to the eye rather than the best fit. The parameters $a$ and $b$ were fixed by eye to be equal to $a=1, b=40$ for $\sigma=0.707$ and $a=0.8, b=73$ for $\sigma=0.576$. A best fit procedure results to be sensitive to the chosen fitting interval and to the tails of the distribution that represent probabilities too small to be correctly estimated with our statistics. Despite these caveats we believe that our data give an indication in favor of the cubic behavior for both values of $\sigma$. 
We then investigated the scaling of the PDF of $u$ with the system size, contrasting it with the behavior of the PDF of $q$. In figures 3 and 5 we display the function $P_{k}(q)$ for the two values of $\sigma$ at low temperatures. The function $P_{k}(q)$ has the characteristic appearance of the one of systems developing RSB for large volumes, with two symmetric peaks and a non-zero part for $q \approx 0$. Figures 4 and 6 in contrast show that the distribution of $u, P_{k}(u)$, is unimodal around zero. Its width is as expected a decreasing function of $k$. In the insets the unscaled data are presented, while the in the main panel the result of scaling the data using the variable $u 2^{\frac{2}{3}(1-\sigma)}$ is shown. We judge the scaling (12) to be very satisfactory, though not perfect for the values of $k$ we explored. Indeed, while for $\sigma=0.576$ we could not find a the value of the ratio $\theta_{q} / b_{q}$ producing a better data collapse than the theoretical value $\frac{2}{3}(1-\sigma)=0.28$, for $\sigma=0.707$ the value

$\theta_{q} / b_{q}=0.22$ produces a better data collapse than the theoretical value $\frac{2}{3}(1-\sigma)=0.19$. We believe that this discrepancy is due to finite size effects, but we can not exclude at present that the theory should be amended in the non-classical region.

Our data show that the best fitting exponent is largely temperature independent in the low temperature region. A direct scaling of the data in the high temperature region for the values of $k=7,8,9,10$ that we dispose, produces an effective exponent that crosses over slowly from the low temperature value towards the paramagnetic value $\theta_{q} / b_{q}=0.5$. We believe that we are seeing a preasymptotic behavior due to the influence of the critical fixed point. This influence could be particularly marked due to the power-law interactions where the critical fixed point continues to attract the system on relatively large scales. Larger and larger values of $k$ are necessary to observe the paramagnetic behavior closer and closer to the critical temperature.

\section{Summary and Conclusion}

In this paper we have proposed a new method to determine numerically the overlap interface exponents first defined in [7. These can be obtained by looking at the finitesize scaling of the PDF of the differences of the overlaps between two replicas in two subsystems. We have applied the definition to hierarchical models, where we could give a theoretical prediction for the scaling of the overlap differences with size. Though the interface exponent is naturally dictated by the model, the dependence in $u$ is found to be nontrivial in presence of RSB. We tested this dependence in numerical simulations finding very satisfactory agreement both in the classical and in the nonclassical region. These results confirm the interest of hierarchical spin-glass models, that combine analytical tractability, nontrivial critical points and RSB low temperature phases.

Acknowledgments It is a pleasure to thank O. C. Martin, M. Mézard, F. RicciTersenghi, P. Contucci, C. Giardinà, C. Giberti and C. Vernia for interesting discussions. 




Figure 3. Probability distribution function $p(q)$ for $\sigma=0.576$ (classical region) and $T=0.745=0.615 T_{c}\left(T_{c}=1.24(1)\right)$ for $L=2^{k}$ with $k=7,8,9,10$. The peaks become sharper and sharper for larger $k$.

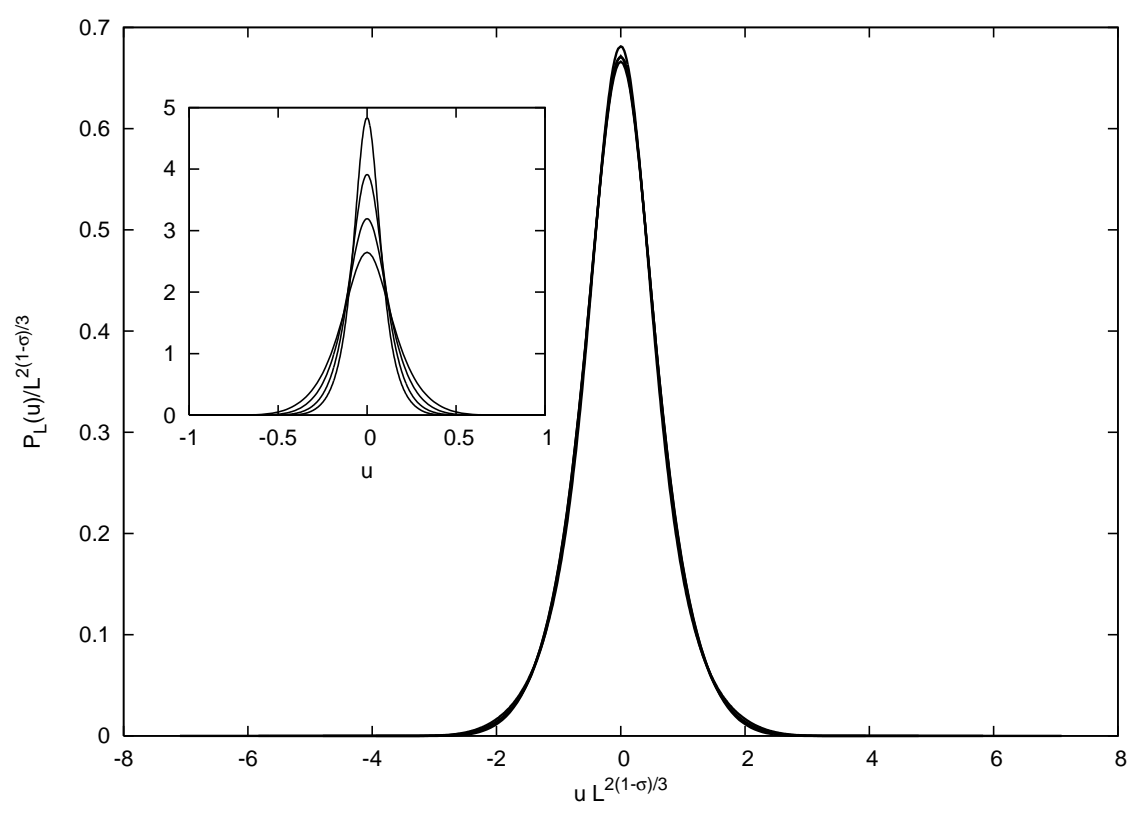

Figure 4. Scaling plot of probability distribution of the overlap difference for $\sigma=0.576$ (classical region) and $T=0.745=0.601 T_{c}$ for $L=2^{k}$ with $k=7,8,9,10$. Inset: the unscaled probability. 


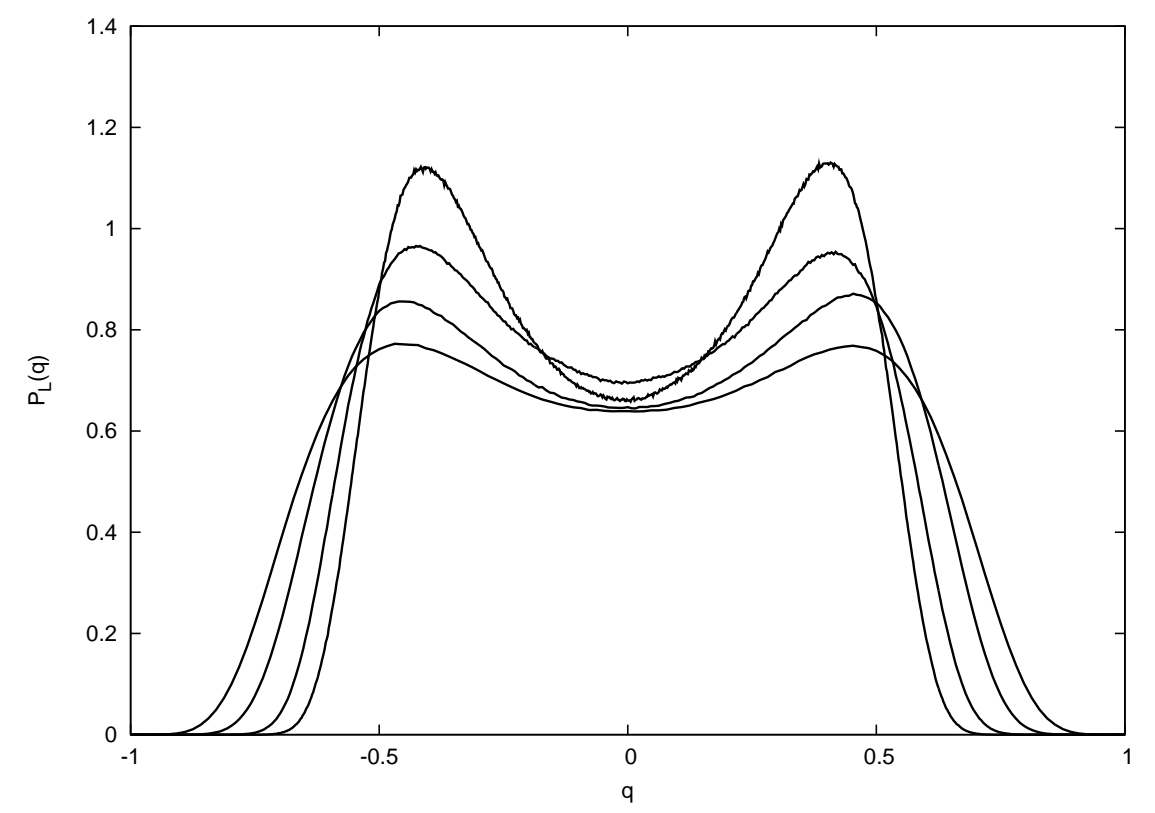

Figure 5. Same as figure 3 for $\sigma=0.707$ in the non-classical region. Here $T_{c}=1.14(1)$ and we present data for $T=0.740=0.65 T_{c}$.

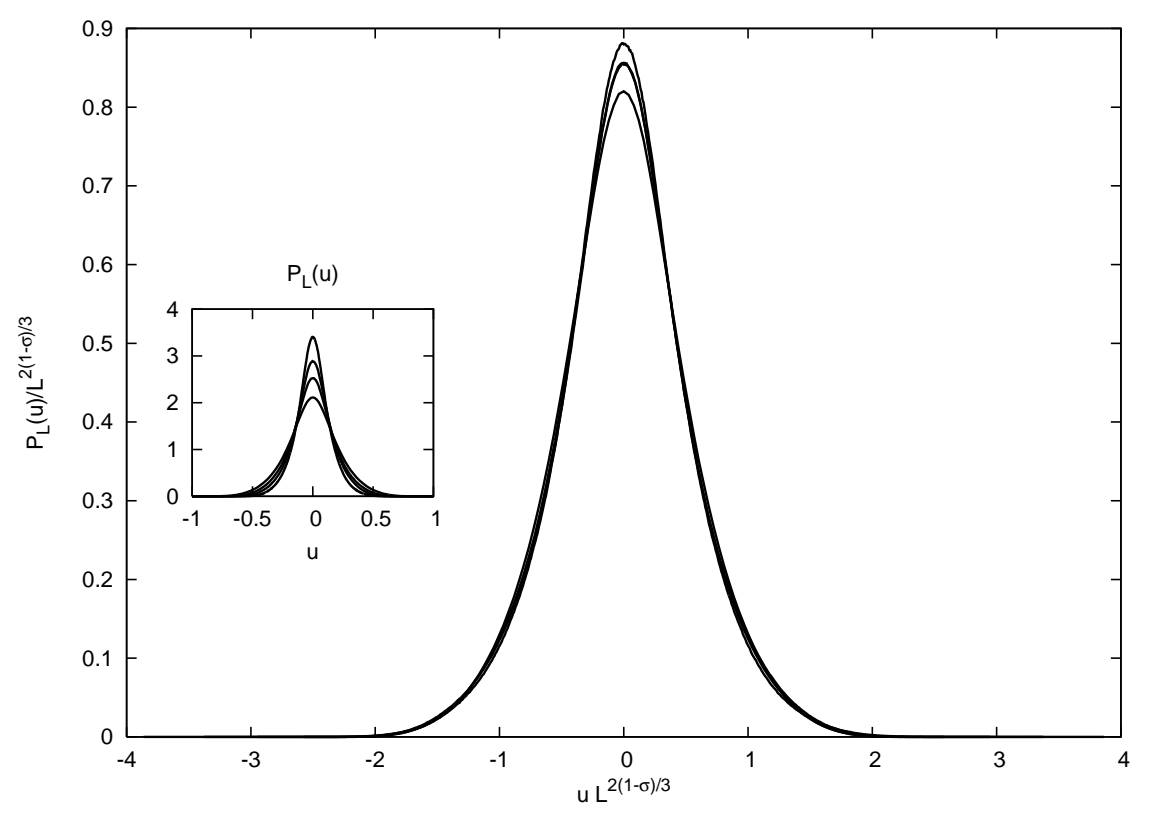

Figure 6. Same as figure 4 for $\sigma=0.707$ in the non-classical region. The temperature is the same as in figure $5, T=0.740$. 


\section{Appendix: Characterization of the critical point.}

In this appendix we discuss the characterization of the critical point of the model for the values of the interaction parameter $\sigma$ that we have considered in the text.

The model has been simulated with the Parallel Tempering algorithm, with 10 values of the temperature, using $2^{20}$ thermalization steps before collecting data in the $2^{20}$ steps. We considered systems sizes of $2^{7}, 2^{8}, 2^{9}$ and $2^{10}$ spins. Averages were performed over 4000 samples for the smaller systems and 700 samples for the largest system. Simulating two replicas in parallel, we have studied the second and the fourth moment of the distribution of the mutual overlap $q_{2}=E\left\langle Q^{2}\right\rangle$ and $q_{4}=E\left\langle Q^{4}\right\rangle$. We have identified the critical temperature and the exponents $\eta$ and $\nu$ using finite size scaling through the

behavior of $q_{2}, q_{4}$ and the corresponding Binder parameter [23] $B=\frac{1}{2}\left(3-\frac{q_{4}}{q_{2}^{2}}\right)$. In the non-classical region, $\sigma>2 / 3$ where finite-size scaling should hold the various parameters exhibit the following dependence on the temperature and system size $L=2^{k}$ :

$$
\begin{aligned}
& \chi=L q_{2}=L^{2-\eta} g_{2}\left(L^{1 / \nu}\left(T-T_{c}\right)\right) \\
& L^{2} q_{4}=L^{4-2 \eta} g_{4}\left(L^{1 / \nu}\left(T-T_{c}\right)\right) \\
& B=b\left(L^{1 / \nu}\left(T-T_{c}\right)\right) .
\end{aligned}
$$

The exponent $\eta$ should not renormalize in long range models, and analogously to the Euclidian 1D model take the value $\eta=3-2 \sigma$ both in the non-classical and in the classical regions [13].

In the classical region, $1 / 2<\sigma<2 / 3$, the scaling implied by (21) does not hold [24, 25]. It is possible to show, using the fact that the critical theory is described by a cubic action analogous to the one for short-range spin glasses [26], that the various quantities scale according to the following:

$$
\begin{aligned}
& L q_{2}=\left|T-T_{c}\right|^{-\gamma} \tilde{g}_{2}\left(L^{1 / 3}\left(T-T_{c}\right)\right) \\
& L^{2} q_{4}=\left|T-T_{c}\right|^{-2 \gamma} \tilde{g}_{4}\left(L^{1 / 3}\left(T-T_{c}\right)\right) \\
& B=\tilde{b}\left(L^{1 / 3}\left(T-T_{c}\right)\right) .
\end{aligned}
$$

The exponent $1 / 3$ in the scaling functions can be derived from dimensional analysis from the cubic action. The exponent $\gamma$ takes the value $\gamma_{c l}=1$ independently of $\sigma$ as can be checked from the scaling relation $\gamma=(2-\eta) \nu$ with $\nu=\frac{1}{2 \sigma-1}$.

Let us now turn to the data considering the non-classical region first. To analyze the data we observe the following procedure: we first estimate the critical temperature from the crossing point of the Binder parameter and the rescaled values, especially, $q_{2} L^{-1+\eta}$ and $q_{4} L^{-2+2 \eta}$ since these provide for a cleaner crossing than the Binder parameter. We then fix the value of $\nu$ in order to collapse the curves. The result for $\sigma=0.707$ is shown in figure 7, we present the data for $q_{2}, q_{4}$ and $B$ scaled as in (22) using a value of $T_{c}=1.14$.

If we try to use the scaling (21) in the classical regime we get inconsistent results: although we obtain an approximate crossing of the curves for the three quantities, the temperatures at which the curves cross do clearly not coincide. The crossing of the 


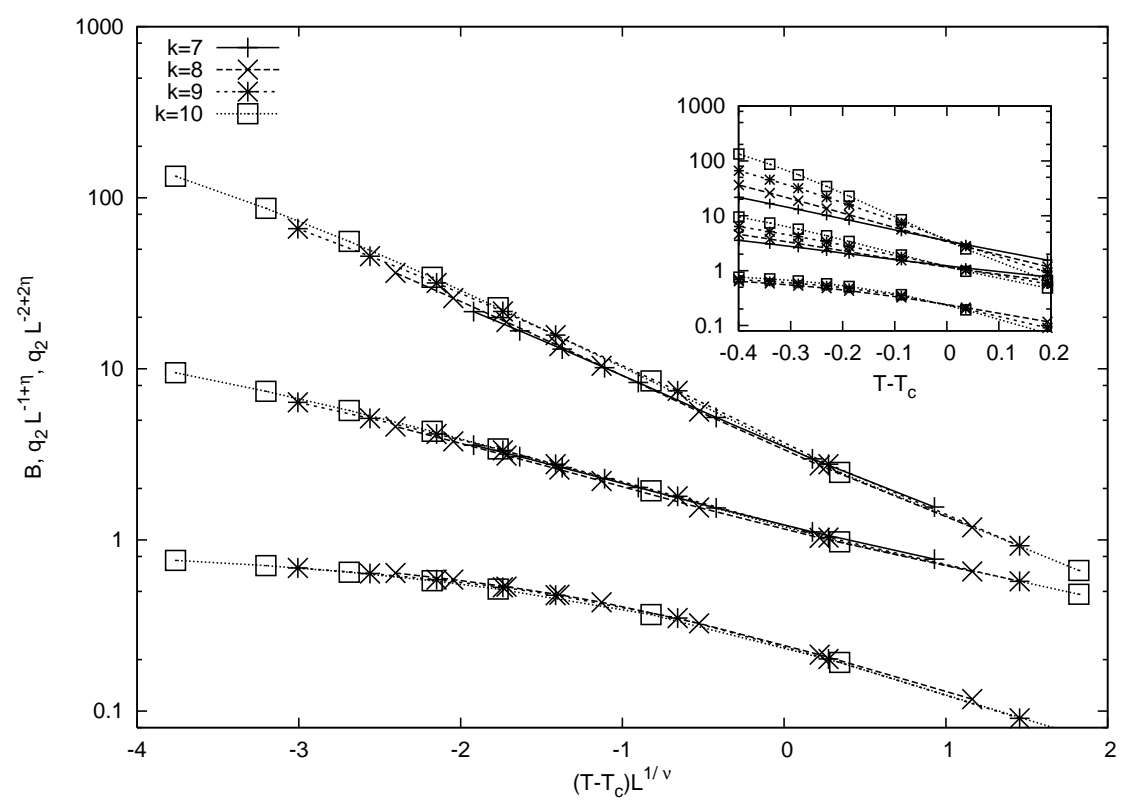

Figure 7. Behavior of $q_{2}, q_{4}$ and $B$ as a function of temperature and system size for $\sigma=0.707$. In the inset we plot $q_{2} / L^{1-\eta}, q_{4} / L^{2-2 \eta}$ and $B$ against $\left(T-T_{c}\right)$, with the values $T_{c}=1.14$ and $\eta=3-2 \sigma$. Main panel, scaling plot of the data $q_{2} / L^{1-\eta}$, $q_{4} / L^{2-2 \eta}$ and $B$ against $\left(T-T_{c}\right) L^{1 / \nu}$ assuming the value of $\nu=3.09$.

Binder parameter indicates $T_{c}=1.24$ and as shown in figure 7 for $\sigma=0.576$, we obtain consistent scaling assuming the form (22) and not (21).

\section{Bibliography}

[1] Zinn-Justin J 2002 Quantum Field Theory and Critical Phenomena (Oxford: Oxford University Press)

[2] Southern B W and Young A P 1977 J. Phys. C: Solid State Phys. 102179

[3] McMillan W L 1984 Phys. Rev. B 29 4026-4029

[4] Bray A J and Moore M A 1984 J. Phys. C: Solid State Phys. 17 L463

[5] Fisher D S and Huse D A 1986 Phys. Rev. Lett. 561601

[6] Boettcher S 2005 Phys. Rev. Lett. 95197205

[7] Franz S, Parisi G and Virasoro M A 1994 J. Phys. I (France) 41657 Preprint cond-mat/9405007

[8] Mézard M, Parisi G and Virasoro M A 1987 Spin Glass Theory and Beyond (Singapore: World Scientific)

[9] Dyson F 1969 Comm. Math. Phys. 1291

[10] Bleher P M and Sinai Ya G 1975 Comm. Math. Phys. 45 247-278

[11] Jona-Lasinio G 1975 Il Nuovo Cimento B 26 99-119

[12] Meurice Y 2007 J. Phys. A: Math. Theo. 40 R39-R102

[13] Kotliar G, Anderson P W and Stein D L 1983 Phys. Rev. B 27602

[14] Leuzzi L 1999 J. Phys. A: Math. Gen. 32 1417-1426

[15] Katzgraber H G and Young A P 2003 Phys. Rev. B 67134410

[16] Katzgraber H G and Young A P 2005 Phys. Rev. B 72184416

[17] Katzgraber H G, Hartmann A K and Young A P 2008 Computer Simulation Studies in Condensed Matter Physics XXI ed D P Landau, S P Lewis and H B Schuttler (Heidelberg, Berlin: Springer Verlag) 


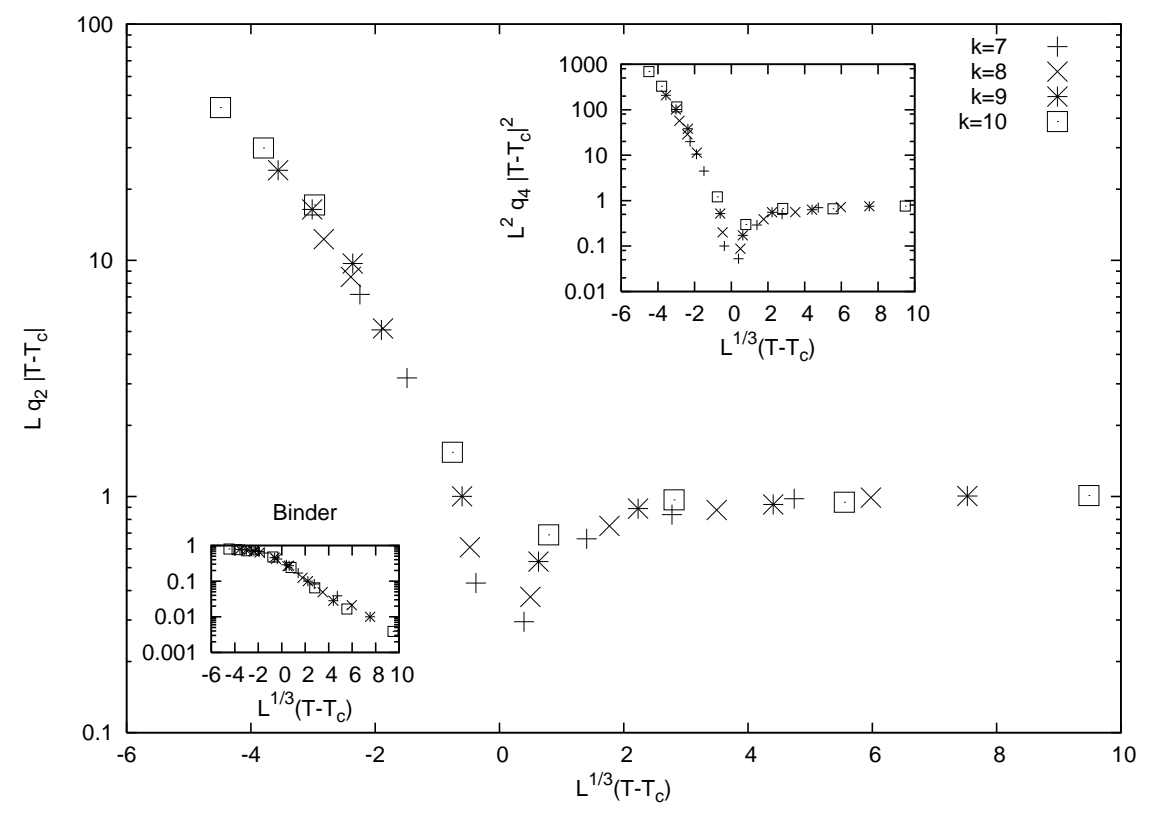

Figure 8. Behavior of $q_{2}, q_{4}$ and $B$ as a function of temperature and system size for $\sigma=0.576$. In the lower inset we show the Binder, for which the data collapse is obtained for $T_{c}=1.14$. The main panel shows $L q_{2}\left|T-T_{C}\right|$. Notice the high temperature behavior corresponding to the Curie law. The curves for larges systems sizes show a tendency of converging toward the value 1 close the critical temperature. In the upper inset we show the scaling for $L^{2} q_{4}\left|T-T_{C}\right|^{2}$ which shows the same qualitative behavior.

[18] Leuzzi L, Parisi G, Ricci-Tersenghi F and Ruiz-Lorenzo J J 2008 Preprint arXiv:0801.4855

[19] Sherrington D and Kirkpatrick S 1975 Phys. Rev. Lett. 351792

[20] Viana L and Bray A J 1985 J. Phys. C: Solid State Phys. 18 3037-51

[21] Franz S and Parisi G 2006 Europhys. Lett. 75385

[22] Franz S, Mézard M, Parisi G and Ricci-Tersenghi F in preparation

[23] Binder K 1981 Z. Phys. B 43119

[24] Brezin E 1982 J. Physique 4315

[25] Brezin E and Zinn-Justin J 1985 Nucl. Phys. B 257867

[26] Harris A B, Lubensky T C and Chen J-H 1976 Phys. Rev. Lett. 36415

[27] Dedominicis C and Kondor I

[28] Contucci P, Giardinà C, Giberti C, Parisi G and Vernia C to be published. 